\title{
Silver-Promoted Oxidative Ring Opening/Alkynylation of Cyclo- propanols: Facile Synthesis of 4-Yn-1-ones
}

\author{
Cheng-Yong Wang \\ Ren-jie Song \\ Ye-Xiang Xie* \\ Jin-Heng Li*
}
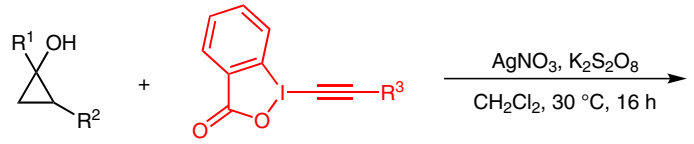

State Key Laboratory of Chemo/Biosensing and Chemometrics,

College of Chemistry and Chemical Engineering, Hunan University,

Changsha 410082, P. R. of China

jhli@hnu.edu.cn

xieyexiang520@126.com

Received: 19.09.2015

Accepted after revision: 16.10.2015

Published online: 17.11 .2015

DOI: 10.1055/s-0035-1560374; Art ID: ss-2015-h0552-op

Abstract A new silver-promoted oxidative ring opening/alkynylation of cyclopropanols with ethynylbenziodoxolones (EBX) is described. This method enables the formation of alkylated alkynes via a sequence of ring opening and alkynylation. Control experiments support a radical mechanism in this silver-promoted method.

Key words silver, ring opening, alkynylation, cyclopropanol, alkyne

Alkynes are common and versatile building blocks with wide application in synthesis. ${ }^{1}$ Therefore, the development of new efficient methods for their synthesis continues to receive the attention of synthetic chemists. ${ }^{1-3}$ Although the Sonogashira cross-coupling reaction, ${ }^{2,3}$ which starts from aryl or alkenyl halides and terminal alkynes, is well-established for the incorporation of alkyne moieties into organic molecules, the synthesis of aliphatic alkynes from alkyl electrophiles remains a formidable challenge. ${ }^{3}$ For example, the Fu group has extended the Sonogashira cross-coupling reaction to the use of primary alkyl halides as the electrophile. ${ }^{3 a}$ The Hu group has also reported an efficient nickelcatalyzed Sonogashira cross-coupling of alkyl halides for the construction of alkylated alkynes. ${ }^{3 b, c}$ However, the majority of these transformations require a copper cocatalyst, base, and a ligand to improve the yield. To overcome these disadvantages, the development of new electrophilic alkynylating reagents, particularly with special reaction characteristics for the formation of the $\mathrm{C}(\mathrm{sp})-\mathrm{C}\left(\mathrm{sp}^{3}\right)$ bond, have gained wide interest in the past decade. ${ }^{4,5}$ Typically, attractive electrophilic alkynylating reagents include ethynylbenziodoxolones (EBX), ${ }^{4}$ which are appealing alkynylating reagents for the construction of diverse ynone molecules by C-alkynylation with aldehydes.

Herein, we report a new oxidative ring opening/alkynylation of cyclopropanols with ethynylbenziodoxolones for the synthesis of alkylated alkynes using a combination of silver(I) nitrate and potassium persulfate as the catalytic system (Scheme 1$) ;{ }^{6}$ this method allows selective radical cleavage of the $\mathrm{C}-\mathrm{C}$ bond in a wide range of cyclopropanols ${ }^{7}$ by various terminal alkynes, including aryl- and alkylsubstituted alkynes, and represents a mild and practical route for the assembly of alkylated alkynes. ${ }^{8}$

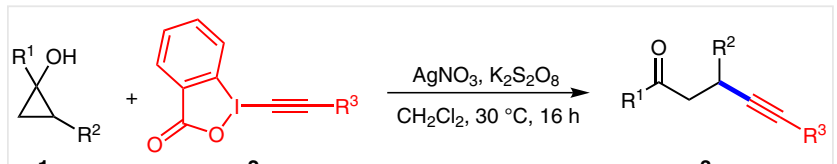

Scheme 1 The ring opening and alkynylation reaction

We began our study by investigating the reaction between 1-(4-methoxyphenyl)cyclopropan-1-ol (1a) and 1(phenylethynyl)-1,2-benziodoxol-3(1H)-one (2a, Ph-EBX) to optimize the reaction conditions (Table 1 ). The results demonstrated that the ring opening/alkynylation reaction occurred in the presence of silver(I) nitrate alone and it enabled the formation of the desired product 3aa in 33\% yield (entry 1). Gratifyingly, the addition of oxidants, such as potassium persulfate, sodium persulfate, ammonium persulfate, and dibenzoyl peroxide (BPO), improved the yield of 3aa (entries 2-5), and potassium persulfate showed the most reactivity. Identical results to those obtained using two equivalents of potassium persulfate were obtained when using three equivalents of potassium persulfate (cf. entries 2 and 6). A number of other silver catalysts (entries 7-10), including silver(I) acetate, tetrafluoroborate, triflate, and carbonate, also had high catalytic activity in this reaction, but they were less effective than silver(I) nitrate. We found that the amount of silver(I) nitrate used also affected 
the reaction result: using $30 \mathrm{~mol} \%$ of silver(I) nitrate did not improve the yield compared to the use of $20 \mathrm{~mol} \%$ of silver(I) nitrate (entry 11 ), but using $10 \mathrm{~mol} \%$ of silver(I) nitrate reduced the yield to $60 \%$ (entry 12 ). Surprisingly, the reaction took place in the absence of a silver salt, albeit with lower yield (45\%) (entry 13). A similar yield (49\%) of 3aa was isolated when three equivalents of potassium persulfate were used in the absence of silver(I) nitrate (entry 14). The results suggest that silver(I) nitrate may play two roles, both as a accelerator and an oxidant. The use of other solvents, dichloromethane-water, 1,2-dichloroethane, acetonitrile, tetrahydrofuran, and $\mathrm{N}, \mathrm{N}$-dimethylformamide, was also examined, but the yields of 3aa were lower than with dichloromethane (entries 15-19). Screening the effects of the reaction temperature revealed that a reaction temperature of $30^{\circ} \mathrm{C}$ gave optimal results (entries 2, 20, and 21).

With the optimal conditions in hand, we set out to investigate the scope and limitations of this oxidative ring opening/alkynylation protocol with regard to cyclopropanols 1 and 1-(substituted ethynyl)-1,2-benziodoxol-3(1H)one 2 (Tables 2 and 3 ). As shown in Table 2, a variety of 1 (arylethynyl)-1,2-benziodoxol-3(1H)-ones $\mathbf{2 b}-\mathbf{h}$ and 1(3,3-dimethylbut-1-ynyl)-1,2-benziodoxol-3(1H)-one (2i) were viable for the construction of the corresponding alkynes 3ab-ai in moderate to good yields, however, 1[(trimethylsilyl)ethynyl]-1,2-benziodoxol-3(1H)-one (2j; TMS-EBX) did not give 3aj. Using 1-(phenylethynyl)-1,2benziodoxol-3(1H)-ones $\mathbf{2 b}$-h, several substituents, such as $\mathrm{Me}, \mathrm{Br}, \mathrm{CN}, \mathrm{Ac}$, and $\mathrm{Ph}$ groups, on the phenyl ring attached to the acetylene were well tolerated giving products $\mathbf{3 a b}$ ah. For example, 1-( $p$-tolylethynyl)-1,2-benziodoxol-3(1H)one (2b) gave 3ab in 70\% yield. 1-(4-Cyanophenylethynyl)1,2-benziodoxol-3 $(1 H)$-one $(\mathbf{2 f})$ and 1-(3-acetylphenylethynyl)-1,2-benziodoxol-3(1H)-one (2g) with a para-cyano or para-acetyl group were also converted into products 3af and 3ag in moderate yields. Importantly, bromo-substituted 1-(phenylethynyl)-1,2-benziodoxol-3(1H)-ones 2c-e utilized under the optimal conditions gave bromo-substituted products 3ac-ae that could undergo subsequent modifications at the halogenated positions. In the case of 1(biphenyl-2-ylethynyl)-1,2-benziodoxol-3(1H)-one (2h) containing an ortho phenyl group the desired product 3ah was obtained in $51 \%$ yield. We found that the optimal conditions were compatible with 1-(3,3-dimethylbut-1-ynyl)1,2-benziodoxol-3(1H)-ones (2i) giving product 3ai in moderate yield.

The optimal conditions were applicable to a wide range of cyclopropanols, namely 1-arylcyclopropanols $\mathbf{1 b}-\mathbf{i}$ and 1-alkylcyclopropanols $\mathbf{1} \mathbf{j}-\mathbf{m}$ (Table 3 ). Initially, a variety of 1 -arylcyclopropanols $\mathbf{1 b}-\mathbf{f}$ were investigated in the presence of 1-(phenylethynyl)-1,2-benziodoxol-3(1H)-one (2a), silver(I) nitrate, and potassium persulfate. We found that
Table 1 Optimization of the Reaction Conditions ${ }^{\mathrm{a}}$

\begin{tabular}{|c|c|c|c|c|}
\hline Entry & {$[\mathrm{Ag}](\mathrm{mol} \%)$} & [O] (equiv) & Solvent & Yield $^{\mathrm{b}}(\%)$ \\
\hline 1 & $\mathrm{AgNO}_{3}(20)$ & - & $\mathrm{CH}_{2} \mathrm{Cl}_{2}$ & 33 \\
\hline 2 & $\mathrm{AgNO}_{3}(20)$ & $\mathrm{K}_{2} \mathrm{~S}_{2} \mathrm{O}_{8}(2)$ & $\mathrm{CH}_{2} \mathrm{Cl}_{2}$ & 81 \\
\hline 3 & $\mathrm{AgNO}_{3}(20)$ & $\mathrm{Na}_{2} \mathrm{~S}_{2} \mathrm{O}_{8}(2)$ & $\mathrm{CH}_{2} \mathrm{Cl}_{2}$ & 73 \\
\hline 4 & $\mathrm{AgNO}_{3}(20)$ & $\left(\mathrm{NH}_{2}\right)_{2} \mathrm{~S}_{2} \mathrm{O}_{8}(2)$ & $\mathrm{CH}_{2} \mathrm{Cl}_{2}$ & 43 \\
\hline 5 & $\mathrm{AgNO}_{3}(20)$ & $\mathrm{BPO}(2)$ & $\mathrm{CH}_{2} \mathrm{Cl}_{2}$ & 65 \\
\hline 6 & $\mathrm{AgNO}_{3}(20)$ & $\mathrm{K}_{2} \mathrm{~S}_{2} \mathrm{O}_{8}$ & $\mathrm{CH}_{2} \mathrm{Cl}_{2}$ & 80 \\
\hline 7 & $\operatorname{AgOAc}(20)$ & $\mathrm{K}_{2} \mathrm{~S}_{2} \mathrm{O}_{8}(2)$ & $\mathrm{CH}_{2} \mathrm{Cl}_{2}$ & 53 \\
\hline 8 & $\mathrm{AgBF}_{4}(20)$ & $\mathrm{K}_{2} \mathrm{~S}_{2} \mathrm{O}_{8}(2)$ & $\mathrm{CH}_{2} \mathrm{Cl}_{2}$ & 20 \\
\hline 9 & $\operatorname{AgOTf}(20)$ & $\mathrm{K}_{2} \mathrm{~S}_{2} \mathrm{O}_{8}(2)$ & $\mathrm{CH}_{2} \mathrm{Cl}_{2}$ & 52 \\
\hline 10 & $\mathrm{Ag}_{2} \mathrm{CO}_{3}(20)$ & $\mathrm{K}_{2} \mathrm{~S}_{2} \mathrm{O}_{8}(2)$ & $\mathrm{CH}_{2} \mathrm{Cl}_{2}$ & 47 \\
\hline 11 & $\mathrm{AgNO}_{3}(30)$ & $\mathrm{K}_{2} \mathrm{~S}_{2} \mathrm{O}_{8}(2)$ & $\mathrm{CH}_{2} \mathrm{Cl}_{2}$ & 78 \\
\hline 12 & $\mathrm{AgNO}_{3}(10)$ & $\mathrm{K}_{2} \mathrm{~S}_{2} \mathrm{O}_{8}(2)$ & $\mathrm{CH}_{2} \mathrm{Cl}_{2}$ & 60 \\
\hline 13 & - & $\mathrm{K}_{2} \mathrm{~S}_{2} \mathrm{O}_{8}(2)$ & $\mathrm{CH}_{2} \mathrm{Cl}_{2}$ & 45 \\
\hline 14 & - & $\mathrm{K}_{2} \mathrm{~S}_{2} \mathrm{O}_{8}(3)$ & $\mathrm{CH}_{2} \mathrm{Cl}_{2}$ & 49 \\
\hline $15^{c}$ & $\mathrm{AgNO}_{3}(20)$ & $\mathrm{K}_{2} \mathrm{~S}_{2} \mathrm{O}_{8}(2)$ & $\mathrm{CH}_{2} \mathrm{Cl}_{2} / \mathrm{H}_{2} \mathrm{O}$ & 50 \\
\hline 16 & $\mathrm{AgNO}_{3}(20)$ & $\mathrm{K}_{2} \mathrm{~S}_{2} \mathrm{O}_{8}(2)$ & DCE & 70 \\
\hline 17 & $\mathrm{AgNO}_{3}(20)$ & $\mathrm{K}_{2} \mathrm{~S}_{2} \mathrm{O}_{8}(2)$ & $\mathrm{MeCN}$ & 19 \\
\hline 18 & $\mathrm{AgNO}_{3}(20)$ & $\mathrm{K}_{2} \mathrm{~S}_{2} \mathrm{O}_{8}(2)$ & THF & 35 \\
\hline 19 & $\mathrm{AgNO}_{3}(20)$ & $\mathrm{K}_{2} \mathrm{~S}_{2} \mathrm{O}_{8}(2)$ & DMF & 61 \\
\hline $20^{d}$ & $\mathrm{AgNO}_{3}(20)$ & $\mathrm{K}_{2} \mathrm{~S}_{2} \mathrm{O}_{8}(2)$ & $\mathrm{CH}_{2} \mathrm{Cl}_{2}$ & 67 \\
\hline $21^{e}$ & $\mathrm{AgNO}_{3}(20)$ & $\mathrm{K}_{2} \mathrm{~S}_{2} \mathrm{O}_{8}(2)$ & $\mathrm{CH}_{2} \mathrm{Cl}_{2}$ & 62 \\
\hline
\end{tabular}

a Reaction conditions: 1 a $(0.3 \mathrm{mmol}), \mathbf{2 a}$ (1.5 equiv), [Ag], oxidant, solvent $(1 \mathrm{~mL}), 30^{\circ} \mathrm{C}$, under argon, $16 \mathrm{~h}$.

${ }^{\mathrm{b}}$ Isolated yield.

${ }^{c} \mathrm{CH}_{2} \mathrm{Cl}_{2}-\mathrm{H}_{2} \mathrm{O}(1: 1)$.

${ }^{\mathrm{C}}$ At r.t.

e At $40{ }^{\circ} \mathrm{C}$.

several substituents, such as $\mathrm{OMe}, \mathrm{Cl}, \mathrm{F}$, and $\mathrm{CF}_{3}$, were tolerated on the phenyl ring. 1-Phenylcyclopropanol (1b) displayed high reactivity and furnished the desired product 3ba in $78 \%$ yield. A substrate containing a bulky ortho group, 2-methoxybenzyl-substituted cyclopropanol 1c, gave 3ca in moderate (60\%) yield. Using 4-chlorophenyl-, 4fluorophenyl-, and 4-(trifluoromethyl)benzyl-substituted cyclopropanols 1d-f gave 3da-fa in $72 \%, 75 \%$, and $53 \%$ yields, respectively. The reaction was applicable to heterocycle-containing substrates $\mathbf{1 g}$ and $\mathbf{1 h}$, and successfully delivered products 3ga and $\mathbf{3 h a}$ in good yields. We were pleased to find that 1-(4-methoxyphenyl)-2-pentylcyclopropan-1-ol (1i) was a suitable substrate and it successfully gave product 3ia. The optimal conditions were compatible with 1-alkylcyclopropanols $\mathbf{1} \mathbf{j}-\mathbf{l}$, even bulky 1-(1-adaman- 
Table 2 Variation of the Ethynylbenziodoxolone $\mathbf{2}^{\mathrm{a}}$

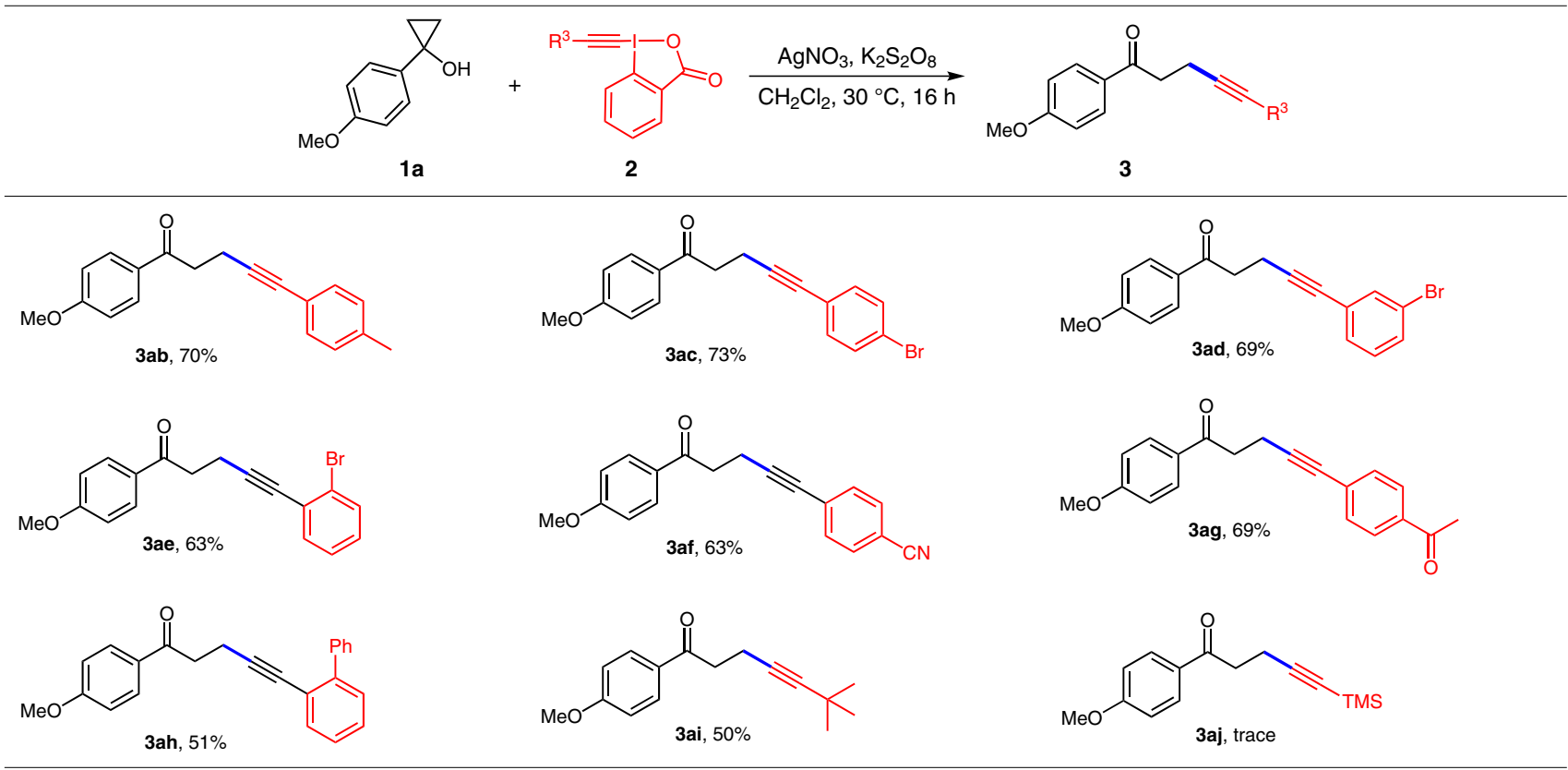

${ }^{a}$ Reaction conditions: $1 \mathrm{a}(0.3 \mathrm{mmol}), 2$ (1.5 equiv), $\mathrm{AgNO}_{3}$ (20 mol\%), $\mathrm{K}_{2} \mathrm{~S}_{2} \mathrm{O}_{8}$ (2 equiv), $\mathrm{CH}_{2} \mathrm{Cl}_{2}(1 \mathrm{~mL}), 30^{\circ} \mathrm{C}$ under argon, $16 \mathrm{~h}$.

tyl)cyclopropan-1-ol (11), affording products 3ja-la in high yields. Gratifyingly, 1-styrylcyclopropan-1-ol (1m) was also a viable substrate for the construction of $3 \mathrm{ma}$ in $55 \%$ yield.

As shown in Scheme 2, the reaction of cyclopropanol 1a with 1-(phenylethynyl)-1,2-benziodoxol-3(1H)-one (2a) was completely suppressed when using a stoichiometric amount of radical inhibitor (3 equiv), including 2,2,6,6-te- tramethylpiperidin-1-oxyl (TEMPO) and 2,6-di-tert-butyl4-methylphenol (BHT). The results suggest that this reaction involves a free radical process.

Therefore, the proposed mechanism outlined in Scheme 2 for this ring opening of cyclopropanols 1 by silver(I) nitrate and potassium persulfate begins by the formation of the alkyl radical intermediate $\mathbf{A}$. The addition of intermedi-

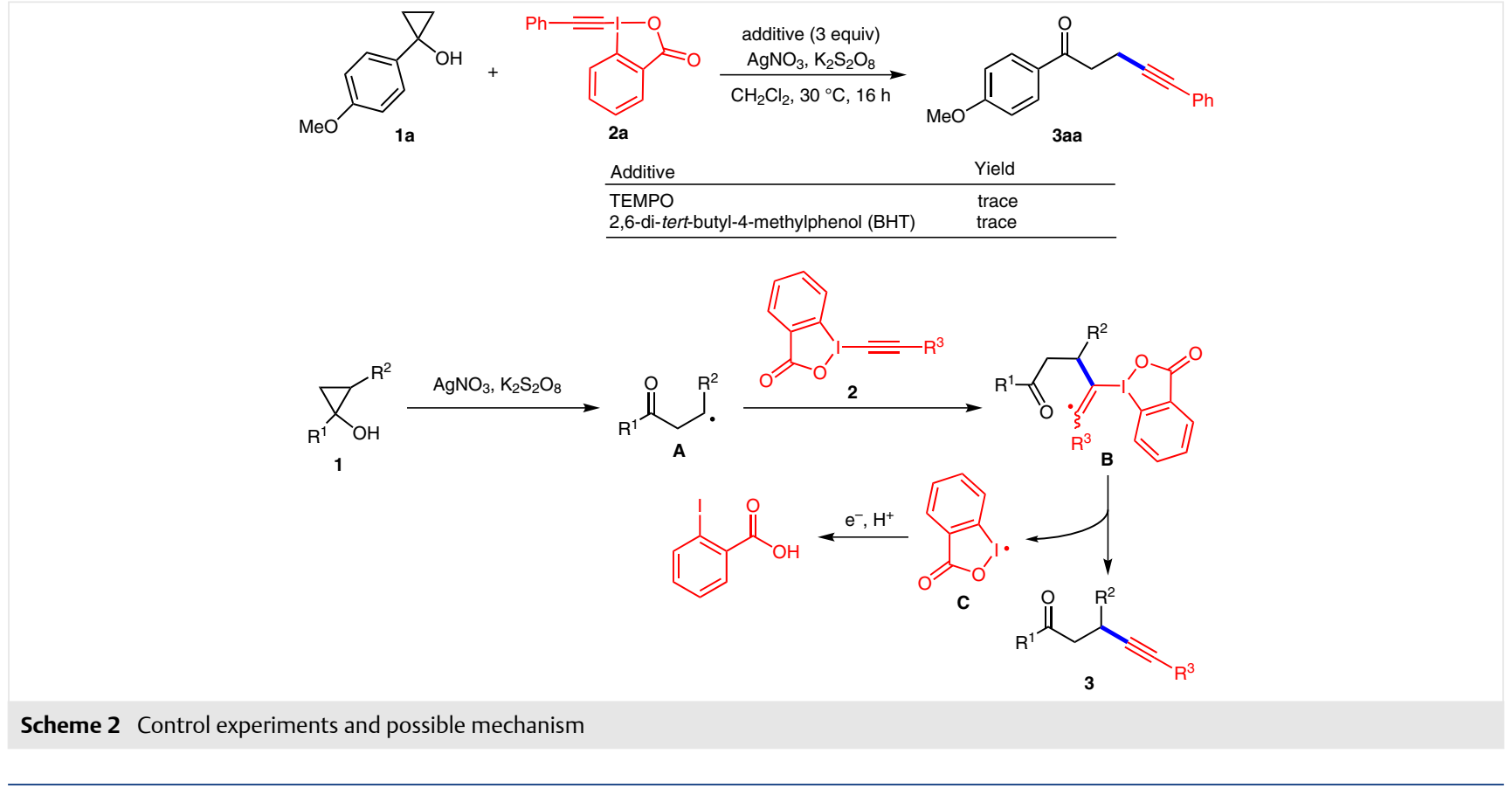


Table 3 Variation of the Cyclopropanol $\mathbf{1}^{\mathrm{a}}$

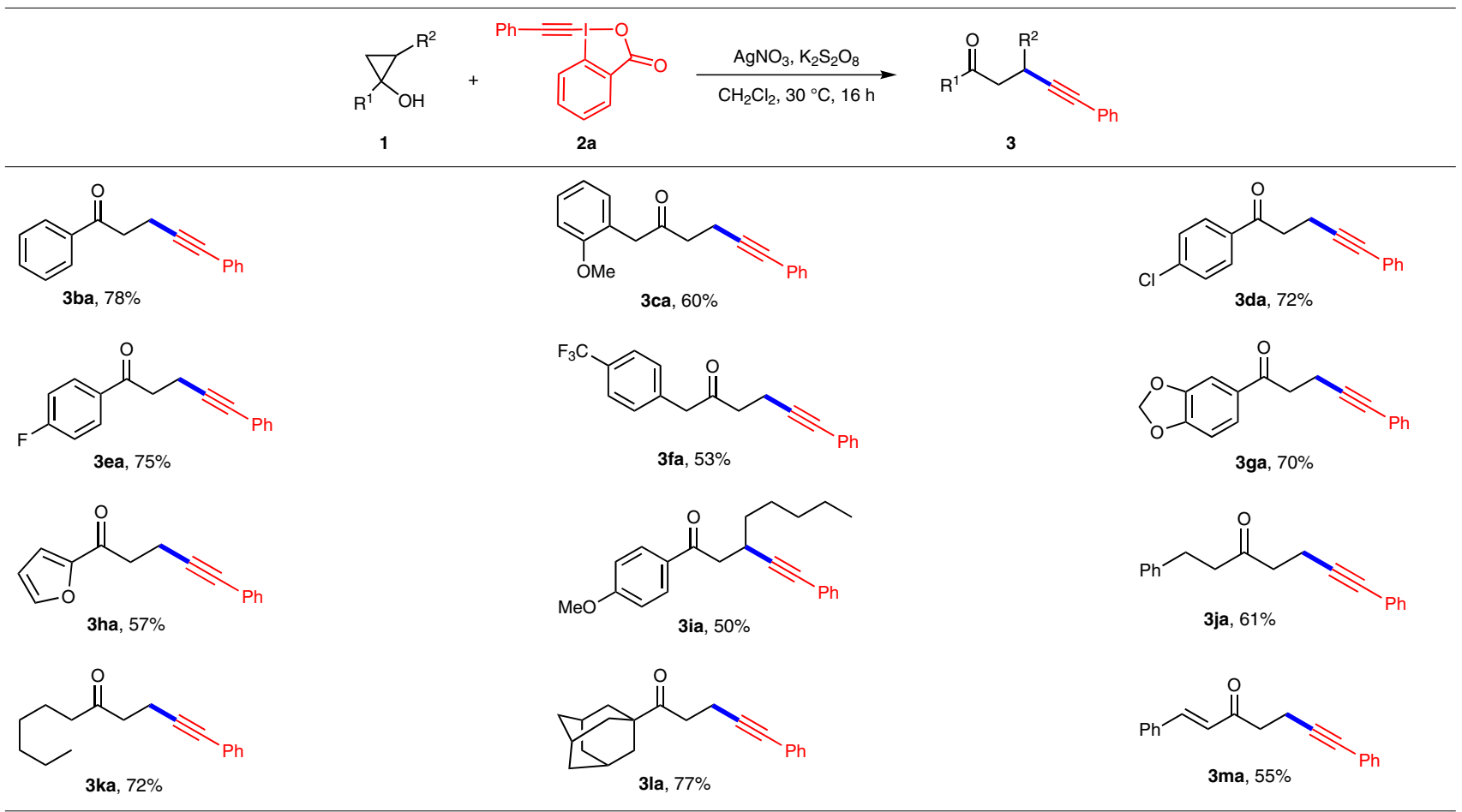

a Reaction conditions: 1 ( $0.3 \mathrm{mmol}$ ), $2 \mathrm{a}$ (1.5 equiv), $\mathrm{AgNO}_{3}$ (20 mol\%), $\mathrm{K}_{2} \mathrm{~S}_{2} \mathrm{O}_{8}$ (2 equiv), $\mathrm{CH}_{2} \mathrm{Cl}_{2}(1 \mathrm{~mL}), 30^{\circ} \mathrm{C}$, under argon, $16 \mathrm{~h}$.

ate $\mathbf{A}$ to the $\mathrm{C} \equiv \mathrm{C}$ bond in $\mathbf{2}$ produces the vinyl radical intermediate $\mathbf{B}$, followed by $\mathrm{C}-\mathrm{I}$ bond cleavage by single-electron transfer and this is followed by $\beta$-elimination to give the desired products $\mathbf{3}$ and radical $\mathbf{C}$. Within this process, silver salts might play at least two roles: as the catalyst to initiate the formation of the radical intermediate $\mathbf{A}$ and as Lewis acid to stabilize the radical intermediates.

In summary, we have developed a new silver-promoted oxidative ring opening/alkynylation of cyclopropanols with ethynylbenziodoxolones for the synthesis of alkylated alkynes in the presence of potassium persulfate. In this method, both silver(I) nitrate and potassium persulfate have two roles as catalysts and oxidants, thus achieving ring opening and alkynylation with broad substrate scope and excellent selectivity.

NMR spectroscopy was performed on a Bruker advanced spectrometer operating at $400 \mathrm{MHz}\left({ }^{1} \mathrm{H} \mathrm{NMR}\right)$ and $100 \mathrm{MHz}\left({ }^{13} \mathrm{C} \mathrm{NMR}\right)$ or 500 $\mathrm{MHz}\left({ }^{1} \mathrm{H}\right.$ NMR) and $125 \mathrm{MHz}\left({ }^{13} \mathrm{C}\right.$ NMR). MS analysis was performed on GC-MS analysis (Shimazu GCMS-QP2010) and ESI-Q-TOF (Bruker MicroQTOF-II). All melting points are uncorrected.

\section{Silver-Promoted Oxidative Ring Opening/Alkynylation of Cyclo- propanols; Typical Procedure}

To a Schlenk tube were added $1(0.3 \mathrm{mmol}), 2(0.45 \mathrm{mmol}), \mathrm{AgNO}_{3}$ (0.06 mmol), $\mathrm{K}_{2} \mathrm{~S}_{2} \mathrm{O}_{8}(0.6 \mathrm{mmol})$, and $\mathrm{CH}_{2} \mathrm{Cl}_{2}(1 \mathrm{~mL})$. The tube was charged with argon ( $1 \mathrm{~atm})$ and stirred at $30{ }^{\circ} \mathrm{C}$ for $16 \mathrm{~h}$ until complete consumption of the starting material (TLC monitoring). When the reaction had finished, the mixture was washed with aq sat. $\mathrm{NaHCO}_{3}$. The aqueous phase was re-extracted with $\mathrm{CH}_{2} \mathrm{Cl}_{2}$. The combined organic extracts were dried $\left(\mathrm{Na}_{2} \mathrm{SO}_{4}\right)$, concentrated under vacuum, and the resulting residue was purified by column chromatography (silica gel, hexane-EtOAc) to afford the desired product.

1-(4-Methoxyphenyl)-5-phenylpent-4-yn-1-one (3aa) ${ }^{9}$

White solid; yield: $64.2 \mathrm{mg}(81 \%)$; $\mathrm{mp} 52.9-54.0^{\circ} \mathrm{C}$.

IR (KBr): $1677 \mathrm{~cm}^{-1}$.

${ }^{1} \mathrm{H} \mathrm{NMR}\left(400 \mathrm{MHz}, \mathrm{CDCl}_{3}\right): \delta=7.96(\mathrm{~d}, J=8.8 \mathrm{~Hz}, 2 \mathrm{H}), 7.39-7.37(\mathrm{~m}, 2$ H), 7.26-7.25 (m, $3 \mathrm{H}), 6.93(\mathrm{~d}, J=8.8 \mathrm{~Hz}, 2 \mathrm{H}), 3.84(\mathrm{~s}, 3 \mathrm{H}), 3.27-3.23$ (m, $2 \mathrm{H}), 2.84-2.81(\mathrm{~m}, 2 \mathrm{H})$.

${ }^{13} \mathrm{C}$ NMR $\left(100 \mathrm{MHz}, \mathrm{CDCl}_{3}\right): \delta=196.4,163.5,131.5,130.2,129.6$, 128.1, 127.6, 123.6, 113.7, 89.0, 80.9, 55.4, 37.4, 14.3 .

LR-MS (EI, $70 \mathrm{eV}): m / z(\%)=264\left(\mathrm{M}^{+}, 37\right), 233$ (18), 221 (18), 135 (100).

HRMS (ESI): $m / z[M+H]^{+}$calcd for $\mathrm{C}_{18} \mathrm{H}_{17} \mathrm{O}_{2}:$ 265.1223; found: 265.1230 .

1-(4-Methoxyphenyl)-5-(p-tolyl)pent-4-yn-1-one (3ab) White solid; yield: $58.4 \mathrm{mg}(70 \%)$; $\mathrm{mp} 72.5-74.2^{\circ} \mathrm{C}$.

IR (KBr): $1675 \mathrm{~cm}^{-1}$.

${ }^{1} \mathrm{H}$ NMR $\left(400 \mathrm{MHz}, \mathrm{CDCl}_{3}\right): \delta=7.97(\mathrm{~d}, J=8.8 \mathrm{~Hz}, 2 \mathrm{H}), 7.27(\mathrm{~d}, J=8.0$ $\mathrm{Hz}, 2 \mathrm{H}), 7.07(\mathrm{~d}, J=8.0 \mathrm{~Hz}, 2 \mathrm{H}), 6.94(\mathrm{~d}, J=8.8 \mathrm{~Hz}, 2 \mathrm{H}), 3.87(\mathrm{~s}, 3 \mathrm{H})$, 3.28-3.24 (m, $2 \mathrm{H}), 2.82(\mathrm{t}, J=8.0 \mathrm{~Hz}, 2 \mathrm{H}), 2.32(\mathrm{~s}, 3 \mathrm{H})$. 
${ }^{13} \mathrm{C}$ NMR $\left(100 \mathrm{MHz}, \mathrm{CDCl}_{3}\right): \delta=196.6,163.5,137.6,131.4,130.2$, $129.7,128.9,120.5,113.7,88.2,81.0,55.4,37.5,21.4,14.4$.

LR-MS (EI, $70 \mathrm{eV}): m / z(\%)=278\left(\mathrm{M}^{+}, 52\right), 235$ (23), 135 (100).

HRMS (ESI): $m / z[M+H]^{+}$calcd for $\mathrm{C}_{19} \mathrm{H}_{19} \mathrm{O}_{2}$ : 279.1380; found: 279.1385 .

5-(4-Bromophenyl)-1-(4-methoxyphenyl)pent-4-yn-1-one (3ac)

White solid; yield: $74.9 \mathrm{mg}(73 \%) ; \mathrm{mp} 112.6-113.7^{\circ} \mathrm{C}$.

IR (KBr): $1683 \mathrm{~cm}^{-1}$.

${ }^{1} \mathrm{H}$ NMR $\left(400 \mathrm{MHz}, \mathrm{CDCl}_{3}\right): \delta=7.97(\mathrm{~d}, J=8.8 \mathrm{~Hz}, 2 \mathrm{H}), 7.39(\mathrm{~d}, J=8.4$ $\mathrm{Hz}, 2 \mathrm{H}), 7.23$ (d, J = 8.4 Hz, $2 \mathrm{H}), 6.94(\mathrm{~d}, J=8.8 \mathrm{~Hz}, 2 \mathrm{H}), 3.87(\mathrm{~s}, 3 \mathrm{H})$, $3.27-3.24(\mathrm{~m}, 2 \mathrm{H}), 2.84-2.80(\mathrm{~m}, 2 \mathrm{H})$.

${ }^{13} \mathrm{C}$ NMR $\left(100 \mathrm{MHz}, \mathrm{CDCl}_{3}\right): \delta=196.3,163.6,133.0,131.4,130.3$, 129.6, 122.6, 121.7, 113.7, 90.4, 79.9, 55.4, 37.2, 14.4 .

LR-MS (EI, $70 \mathrm{eV}): m / z(\%)=344\left(\mathrm{M}^{+}+2,20\right), 342\left(\mathrm{M}^{+}, 18\right), 313(10)$, 311 (9), 135 (100).

HRMS (ESI): $m / z[\mathrm{M}+\mathrm{H}]^{+}$calcd for $\mathrm{C}_{18} \mathrm{H}_{16} \mathrm{BrO}_{2}$ : 343.0328; found: 343.0335 .

5-(3-Bromophenyl)-1-(4-methoxyphenyl)pent-4-yn-1-one (3ad) White solid; yield: $70.8 \mathrm{mg}(69 \%) ; \mathrm{mp} 78.2-79.5^{\circ} \mathrm{C}$.

IR (KBr): $1678 \mathrm{~cm}^{-1}$.

${ }^{1} \mathrm{H} \mathrm{NMR}\left(400 \mathrm{MHz}, \mathrm{CDCl}_{3}\right): \delta=7.97(\mathrm{~d}, J=8.8 \mathrm{~Hz}, 2 \mathrm{H}), 7.51(\mathrm{~s}, 1 \mathrm{H})$, $7.39(\mathrm{~d}, J=8.0 \mathrm{~Hz}, 1 \mathrm{H}), 7.29(\mathrm{~d}, J=8.0 \mathrm{~Hz}, 1 \mathrm{H}), 7.13(\mathrm{t}, J=8.0 \mathrm{~Hz}, 1 \mathrm{H})$, $6.95(\mathrm{~d}, J=8.8 \mathrm{~Hz}, 2 \mathrm{H}), 3.87(\mathrm{~s}, 3 \mathrm{H}), 3.26(\mathrm{t}, J=7.6 \mathrm{~Hz}, 2 \mathrm{H}), 2.83(\mathrm{t}$, $J=7.2 \mathrm{~Hz}, 2 \mathrm{H}$ ).

${ }^{13} \mathrm{C}$ NMR $\left(100 \mathrm{MHz}, \mathrm{CDCl}_{3}\right): \delta=196.3,163.6,134.4,130.8,130.3$, 130.1, 129.6 (2 C), 125.7, 122.0, 113.8, 90.6, 79.6, 55.5, 37.2, 14.4 .

LR-MS (EI, $70 \mathrm{eV}): m / z(\%)=344\left(\mathrm{M}^{+}+2,15\right), 342\left(\mathrm{M}^{+}, 15\right), 313(11)$, $311(10), 135$ (100).

HRMS (ESI): $m / z[\mathrm{M}+\mathrm{H}]^{+}$calcd for $\mathrm{C}_{18} \mathrm{H}_{16} \mathrm{BrO}_{2}$ : 343.0328; found: 343.0336 .

\section{5-(2-Bromophenyl)-1-(4-methoxyphenyl)pent-4-yn-1-one (3ae)}

White solid; yield: $64.6 \mathrm{mg}(63 \%) ; \mathrm{mp} 83.6-85.2^{\circ} \mathrm{C}$.

IR (KBr): $1671 \mathrm{~cm}^{-1}$.

${ }^{1} \mathrm{H} \mathrm{NMR}\left(400 \mathrm{MHz}, \mathrm{CDCl}_{3}\right): \delta=7.98(\mathrm{~d}, J=8.8 \mathrm{~Hz}, 2 \mathrm{H}), 7.54(\mathrm{~d}, J=8.0$ $\mathrm{Hz}, 1 \mathrm{H}), 7.41(\mathrm{~d}, J=7.6 \mathrm{~Hz}, 1 \mathrm{H}), 7.21(\mathrm{t}, J=7.2 \mathrm{~Hz}, 1 \mathrm{H}), 7.11(\mathrm{t}, J=7.6$ $\mathrm{Hz}, 1 \mathrm{H}), 6.94(\mathrm{~d}, J=8.4 \mathrm{~Hz}, 2 \mathrm{H}), 3.87(\mathrm{~s}, 3 \mathrm{H}), 3.31(\mathrm{t}, J=7.6 \mathrm{~Hz}, 2 \mathrm{H})$, 2.92-2.88 (m, $2 \mathrm{H})$.

${ }^{13} \mathrm{C}$ NMR $\left(100 \mathrm{MHz}, \mathrm{CDCl}_{3}\right): \delta=196.3,163.6,133.3,132.2,130.3$, 129.6, 128.8, 126.9, 125.7, 125.4, 113.7, 94.2, 79.7, 55.4, 37.2, 14.6 .

LR-MS (EI, $70 \mathrm{eV}): m / z(\%)=344\left(\mathrm{M}^{+}+2,4\right), 342\left(\mathrm{M}^{+}, 4\right), 264(20), 263$ (100), 135 (95).

HRMS (ESI): $m / z[\mathrm{M}+\mathrm{H}]^{+}$calcd for $\mathrm{C}_{18} \mathrm{H}_{16} \mathrm{BrO}_{2}$ : 343.0328; found: 343.0333.

4-[5-(4-Methoxyphenyl)-5-oxopent-1-ynyl]benzonitrile (3af) White solid; yield: $54.6 \mathrm{mg}(63 \%) ; \mathrm{mp} 108.3-109.5{ }^{\circ} \mathrm{C}$.

IR (KBr): $1669 \mathrm{~cm}^{-1}$.

${ }^{1} \mathrm{H} \mathrm{NMR}\left(400 \mathrm{MHz}, \mathrm{CDCl}_{3}\right): \delta=7.98(\mathrm{~d}, J=8.8 \mathrm{~Hz}, 2 \mathrm{H}), 7.56(\mathrm{~d}, J=8.4$ $\mathrm{Hz}, 2 \mathrm{H}), 7.45(\mathrm{~d}, J=8.0 \mathrm{~Hz}, 2 \mathrm{H}), 6.96(\mathrm{~d}, J=8.8 \mathrm{~Hz}, 2 \mathrm{H}), 3.88(\mathrm{~s}, 3 \mathrm{H})$, $3.28(\mathrm{t}, J=7.2 \mathrm{~Hz}, 2 \mathrm{H}), 2.87(\mathrm{t}, J=7.2 \mathrm{~Hz}, 2 \mathrm{H})$.

${ }^{13} \mathrm{C}$ NMR $\left(100 \mathrm{MHz}, \mathrm{CDCl}_{3}\right): \delta=196.1,163.7,132.1,131.9,130.3$, 129.5, 128.7, 118.6, 113.8, 111.0, 94.2, 79.7, 55.5, 37.0, 14.0.
LR-MS (EI, $70 \mathrm{eV}): m / z(\%)=289\left(\mathrm{M}^{+}, 30\right), 288$ (23), 258 (29), 135 (100).

HRMS (ESI): $m / z[M+\mathrm{H}]^{+}$calcd for $\mathrm{C}_{19} \mathrm{H}_{16} \mathrm{NO}_{2}$ : 290.1176; found: 290.1186.

5-(4-Acetylphenyl)-1-(4-methoxyphenyl)pent-4-yn-1-one (3ag)

Light yellow solid; yield: $63.4 \mathrm{mg}(69 \%) ; \mathrm{mp} 56.6-57.8^{\circ} \mathrm{C}$.

IR (KBr): $1685,1600 \mathrm{~cm}^{-1}$.

${ }^{1} \mathrm{H} \mathrm{NMR}\left(400 \mathrm{MHz}, \mathrm{CDCl}_{3}\right): \delta=7.99(\mathrm{~d}, J=8.8 \mathrm{~Hz}, 2 \mathrm{H}), 7.70(\mathrm{~d}, J=8.0$ $\mathrm{Hz}, 2 \mathrm{H}), 7.45$ (d, J = 8.4 Hz, $2 \mathrm{H}), 6.95$ (d, $J=8.8 \mathrm{~Hz}, 2 \mathrm{H}), 3.88(\mathrm{~s}, 3 \mathrm{H})$, 3.31-3.27 (m, $2 \mathrm{H}), 2.87(\mathrm{t}, J=7.2 \mathrm{~Hz}, 2 \mathrm{H}), 2.59(\mathrm{~s}, 3 \mathrm{H})$.

${ }^{13} \mathrm{C}$ NMR $\left(100 \mathrm{MHz}, \mathrm{CDCl}_{3}\right): \delta=197.4,196.3,163.7,135.8,131.7$, 130.3, 129.6, 128.7, 128.1, 113.8, 92.9, 80.5, 55.5, 37.2, 26.6, 14.5.

LR-MS (EI, $70 \mathrm{eV}): m / z(\%)=306\left(\mathrm{M}^{+}, 27\right), 291(28), 263$ (12), 135 (100).

HRMS (ESI): $m / z[M+H]^{+}$calcd for $\mathrm{C}_{20} \mathrm{H}_{19} \mathrm{O}_{3}$ : 307.1329; found: 307.1335.

\section{5-(Biphenyl-2-yl)-1-(4-methoxyphenyl)pent-4-yn-1-one (3ah)}

Yellow liquid; yield: $52.0 \mathrm{mg}$ (51\%).

IR (KBr): $1684 \mathrm{~cm}^{-1}$.

${ }^{1} \mathrm{H} \mathrm{NMR}\left(400 \mathrm{MHz}, \mathrm{CDCl}_{3}\right): \delta=7.91(\mathrm{~d}, J=9.2 \mathrm{~Hz}, 2 \mathrm{H}), 7.56(\mathrm{~d}, J=7.2$ $\mathrm{Hz}, 2 \mathrm{H}), 7.49$ (d, J = 7.6 Hz, $1 \mathrm{H}), 7.38-7.29(\mathrm{~m}, 5 \mathrm{H}), 7.28-7.24(\mathrm{~m}, 1$ $\mathrm{H}), 6.94(\mathrm{~d}, J=8.8 \mathrm{~Hz}, 2 \mathrm{H}), 3.88(\mathrm{~s}, 3 \mathrm{H}), 3.10-3.06(\mathrm{~m}, 2 \mathrm{H}), 2.73-2.69$ ( $\mathrm{m}, 2 \mathrm{H})$.

${ }^{13} \mathrm{C}$ NMR $\left(100 \mathrm{MHz}, \mathrm{CDCl}_{3}\right): \delta=196.5,163.6,143.7,140.8,132.9$, 130.3, 129.7, 129.3 (2C), 127.9, 127.8, 127.2, 126.9, 122.0, 113.7, 92.1, $80.6,55.5,37.1,14.5$.

LR-MS (EI, $70 \mathrm{eV}): m / z(\%)=340\left(\mathrm{M}^{+}, 12\right), 339(14), 135$ (100).

HRMS (ESI): $m / z[\mathrm{M}+\mathrm{H}]^{+}$calcd for $\mathrm{C}_{24} \mathrm{H}_{21} \mathrm{O}_{2}$ : 341.1536; found: 341.1538 .

\section{1-(4-Methoxyphenyl)-6,6-dimethylhept-4-yn-1-one (3ai)}

Colorless liquid; yield: $36.7 \mathrm{mg}$ (50\%).

IR (KBr): $1711 \mathrm{~cm}^{-1}$.

${ }^{1} \mathrm{H}$ NMR (400 MHz, $\left.\mathrm{CDCl}_{3}\right): \delta=7.96(\mathrm{~d}, J=8.8 \mathrm{~Hz}, 2 \mathrm{H}), 6.94(\mathrm{~d}, J=8.8$ $\mathrm{Hz}, 2 \mathrm{H}), 3.87$ (s, $3 \mathrm{H}), 3.14-3.10$ (m, $2 \mathrm{H}), 2.58-2.55$ (m, $2 \mathrm{H}), 1.17$ (s, 9 $\mathrm{H})$.

${ }^{13} \mathrm{C} \mathrm{NMR}\left(100 \mathrm{MHz}, \mathrm{CDCl}_{3}\right): \delta=197.2,163.5,130.4,130.2,113.7,89.5$, 77.2, 55.5, 38.0, 31.3, 27.3, 14.0.

LR-MS (EI, $70 \mathrm{eV}): m / z(\%)=244\left(\mathrm{M}^{+}, 7\right), 229(21), 135$ (100).

HRMS (ESI): $m / z[M+H]^{+}$calcd for $\mathrm{C}_{16} \mathrm{H}_{21} \mathrm{O}_{2}$ : 245.1536; found: 245.1541 .

\section{1,5-Diphenylpent-4-yn-1-one (3ba) ${ }^{8 \mathrm{c}}$}

White solid; yield: $54.8 \mathrm{mg}$ (78\%); $\mathrm{mp} 57.7-58.7^{\circ} \mathrm{C}$.

IR (KBr): $1685 \mathrm{~cm}^{-1}$.

${ }^{1} \mathrm{H}$ NMR $\left(400 \mathrm{MHz}, \mathrm{CDCl}_{3}\right): \delta=7.99(\mathrm{~d}, J=8.0 \mathrm{~Hz}, 2 \mathrm{H}), 7.57(\mathrm{t}, J=7.2$ $\mathrm{Hz}, 1 \mathrm{H}), 7.47$ (t, J = 7.6 Hz, $2 \mathrm{H}$ ), 7.39-7.37 (m, $2 \mathrm{H}), 7.27-7.25$ (m, 3 $\mathrm{H}), 3.31(\mathrm{t}, J=7.2 \mathrm{~Hz}, 2 \mathrm{H}), 2.85(\mathrm{t}, J=7.2 \mathrm{~Hz}, 2 \mathrm{H})$.

${ }^{13} \mathrm{C}$ NMR $\left(100 \mathrm{MHz}, \mathrm{CDCl}_{3}\right): \delta=197.9,136.5,133.2,131.5,128.6$, $128.1,128.0,127.7,123.6,88.8,81.0,37.8,14.3$.

LR-MS (EI, $70 \mathrm{eV}): m / z(\%)=234\left(\mathrm{M}^{+}, 49\right), 233(64), 128(32), 105$ (100), 77 (73). 
HRMS (ESI): $m / z[M+H]^{+}$calcd for $\mathrm{C}_{17} \mathrm{H}_{15} \mathrm{O}$ : 235.1117; found: 235.1124.

\section{1-(2-Methoxyphenyl)-6-phenylhex-5-yn-2-one (3ca)}

Light yellow liquid; yield: $50.1 \mathrm{mg}(60 \%)$.

IR (KBr): $1683 \mathrm{~cm}^{-1}$.

${ }^{1} \mathrm{H} \mathrm{NMR}\left(400 \mathrm{MHz}, \mathrm{CDCl}_{3}\right): \delta=7.37-7.35(\mathrm{~m}, 2 \mathrm{H}), 7.27-7.25(\mathrm{~m}, 4 \mathrm{H})$, $7.14(\mathrm{t}, J=7.2 \mathrm{~Hz}, 1 \mathrm{H}), 6.94-6.87(\mathrm{~m}, 2 \mathrm{H}), 3.80(\mathrm{~s}, 3 \mathrm{H}), 3.71(\mathrm{~s}, 2 \mathrm{H})$, 2.78-2.74 (m, $2 \mathrm{H}), 2.67-2.63(\mathrm{~m}, 2 \mathrm{H})$.

${ }^{13} \mathrm{C}$ NMR $\left(100 \mathrm{MHz}, \mathrm{CDCl}_{3}\right): \delta=206.8,157.3,131.5,131.2,128.6$, 128.2, 127.7, 123.6, 123.2, 120.7, 110.5, 88.8, 80.8, 55.3, 44.7, 40.7, 14.0.

LR-MS (EI, 70 eV): $m / z(\%)=278\left(\mathrm{M}^{+}, 23\right), 157$ (82), 115 (100), 91 (91). HRMS (ESI): $m / z[M+H]^{+}$calcd for $\mathrm{C}_{19} \mathrm{H}_{19} \mathrm{O}_{2}$ : 279.1380; found: 279.1384 .

\section{1-(4-Chlorophenyl)-5-phenylpent-4-yn-1-one (3da) ${ }^{9}$}

White solid; yield: $57.9 \mathrm{mg}(72 \%) ; \mathrm{mp} 49.8-52.4{ }^{\circ} \mathrm{C}$.

IR (KBr): $1688 \mathrm{~cm}^{-1}$.

${ }^{1} \mathrm{H}$ NMR $\left(400 \mathrm{MHz}, \mathrm{CDCl}_{3}\right): \delta=7.93(\mathrm{~d}, J=8.4 \mathrm{~Hz}, 2 \mathrm{H}), 7.45(\mathrm{~d}, J=8.4$ $\mathrm{Hz}, 2 \mathrm{H}), 7.38-7.36$ ( $\mathrm{m}, 2 \mathrm{H}), 7.28-7.26$ (m, $3 \mathrm{H}), 3.29$ (t, $J=7.6 \mathrm{~Hz}, 2$ $\mathrm{H}), 2.85(\mathrm{t}, J=7.2 \mathrm{~Hz}, 2 \mathrm{H})$.

${ }^{13} \mathrm{C}$ NMR $\left(100 \mathrm{MHz}, \mathrm{CDCl}_{3}\right): \delta=196.8,139.7,134.9,131.5,129.5$, 129.0, 128.2, 127.8, 123.5, 88.5, 81.2, 37.8, 14.3.

LR-MS (EI, $70 \mathrm{eV}): m / z(\%)=270\left(\mathrm{M}^{+}+2,15\right), 268\left(\mathrm{M}^{+}, 45\right), 233(49)$, $111(52)$.

HRMS (ESI): $m / z[\mathrm{M}+\mathrm{H}]^{+}$calcd for $\mathrm{C}_{17} \mathrm{H}_{14} \mathrm{ClO}$ : 269.0728; found: 269.0736 .

\section{1-(4-Fluorophenyl)-5-phenylpent-4-yn-1-one (3ea) ${ }^{9}$}

Colorless liquid; yield: $56.7 \mathrm{mg}$ (75\%).

IR (KBr): $1690 \mathrm{~cm}^{-1}$.

${ }^{1} \mathrm{H}$ NMR $\left(400 \mathrm{MHz}, \mathrm{CDCl}_{3}\right): \delta=8.04-8.00(\mathrm{~m}, 2 \mathrm{H}), 7.37(\mathrm{~d}, J=3.6 \mathrm{~Hz}, 2$ H), $7.27(\mathrm{~d}, J=3.2 \mathrm{~Hz}, 3 \mathrm{H}), 7.14(\mathrm{t}, J=8.4 \mathrm{~Hz}, 2 \mathrm{H}), 3.29(\mathrm{t}, J=8.0 \mathrm{~Hz}, 2$ $\mathrm{H}), 2.85(\mathrm{t}, J=7.2 \mathrm{~Hz}, 2 \mathrm{H})$.

${ }^{13} \mathrm{C}$ NMR $\left(100 \mathrm{MHz}, \mathrm{CDCl}_{3}\right): \delta=196.4,166.3\left(\mathrm{~d}, J_{\mathrm{C}-\mathrm{F}}=253.0 \mathrm{~Hz}\right), 133.0$ $\left(d, J_{C-F}=2.0 \mathrm{~Hz}\right), 131.5,130.7\left(\mathrm{~d}, J_{\mathrm{C}-\mathrm{F}}=9.0 \mathrm{~Hz}\right), 128.2,127.7,123.5$, $115.7\left(\mathrm{~d}, J_{\mathrm{C}-\mathrm{F}}=21.0 \mathrm{~Hz}\right), 88.6,81.1,37.7,14.3$.

${ }^{19} \mathrm{~F}$ NMR $\left(375 \mathrm{MHz}, \mathrm{CDCl}_{3}\right): \delta=-104.9$.

LR-MS (EI, $70 \mathrm{eV}): m / z(\%)=252\left(\mathrm{M}^{+}, 58\right), 251$ (78), 209 (20), 128 (27), 123 (100), 95 (57).

HRMS (ESI): $m / z[M+H]^{+}$calcd for $\mathrm{C}_{17} \mathrm{H}_{14} \mathrm{FO}$ : 253.1023; found: 253.1029.

\section{6-Phenyl-1-[4-(trifluoromethyl)phenyl]hex-5-yn-2-one (3fa)}

Light yellow solid; yield: $50.3 \mathrm{mg}(53 \%)$; $\mathrm{mp} 50.6-52.4{ }^{\circ} \mathrm{C}$.

IR (KBr): $1669 \mathrm{~cm}^{-1}$.

${ }^{1} \mathrm{H} \mathrm{NMR}\left(400 \mathrm{MHz}, \mathrm{CDCl}_{3}\right): \delta=7.58(\mathrm{~d}, J=8.0 \mathrm{~Hz}, 2 \mathrm{H}), 7.35-7.32(\mathrm{~m}, 4$ H), 7.28-7.25 (m, 3 H), 3.82 (s, 2 H), 2.82-2.78 (m, 2 H), 2.69-2.65 (m, $2 \mathrm{H})$.

${ }^{13} \mathrm{C}$ NMR $\left(100 \mathrm{MHz}, \mathrm{CDCl}_{3}\right): \delta=205.1,137.7,131.5,129.8,129.5$ (q, $\left.J_{\mathrm{C}-\mathrm{F}}=32.3 \mathrm{~Hz}\right), 128.2,127.8,125.6\left(\mathrm{q}, J_{\mathrm{C}-\mathrm{F}}=3.8 \mathrm{~Hz}\right), 123.8\left(\mathrm{q}, J_{\mathrm{C}-\mathrm{F}}=\right.$ $251.0 \mathrm{~Hz}), 123.4,88.1,81.2,49.6,41.2,14.0$.

${ }^{19} \mathrm{~F} \mathrm{NMR}\left(375 \mathrm{MHz}, \mathrm{CDCl}_{3}\right): \delta=-62.5$.

LR-MS (EI, $70 \mathrm{eV}): m / z(\%)=316\left(\mathrm{M}^{+}, 7\right), 157$ (73), $128(25), 115$ (100).
HRMS (ESI): $m / z[\mathrm{M}+\mathrm{H}]^{+}$calcd for $\mathrm{C}_{19} \mathrm{H}_{16} \mathrm{~F}_{3} \mathrm{O}$ : 317.1148; found: 317.1143.

1-(1,3-Benzodioxol-5-yl)-5-phenylpent-4-yn-1-one (3ga)

White solid; yield: $58.4 \mathrm{mg}$ (70\%); $\mathrm{mp} 79.6-82.3^{\circ} \mathrm{C}$.

IR (KBr): $1674 \mathrm{~cm}^{-1}$.

${ }^{1} \mathrm{H} \mathrm{NMR}\left(400 \mathrm{MHz}, \mathrm{CDCl}_{3}\right): \delta=7.60-7.58(\mathrm{~m}, 1 \mathrm{H}), 7.46(\mathrm{~d}, J=1.2 \mathrm{~Hz}, 1$ $\mathrm{H}), 7.39-7.37(\mathrm{~m}, 2 \mathrm{H}), 7.27-7.26(\mathrm{~m}, 3 \mathrm{H}), 6.85(\mathrm{~d}, J=8.0 \mathrm{~Hz}, 1 \mathrm{H})$, $6.03(\mathrm{~s}, 2 \mathrm{H}), 3.23(\mathrm{t}, J=7.2 \mathrm{~Hz}, 2 \mathrm{H}), 2.82(\mathrm{t}, J=7.2 \mathrm{~Hz}, 2 \mathrm{H})$.

${ }^{13} \mathrm{C}$ NMR $\left(100 \mathrm{MHz}, \mathrm{CDCl}_{3}\right): \delta=196.0,151.8,148.2,131.5,131.4$, 128.1, 127.7, 124.3, 123.6, 107.9, 107.8, 101.8, 88.9, 81.0, 37.5, 14.4 .

LR-MS (EI, $70 \mathrm{eV}): m / z(\%)=278\left(\mathrm{M}^{+}, 53\right), 277$ (41), 235 (20), 149 (100).

HRMS (ESI): $m / z[M+H]^{+}$calcd for $\mathrm{C}_{18} \mathrm{H}_{15} \mathrm{O}_{3}$ : 279.1016; found: 279.1022 .

1-(Furan-2-yl)-5-phenylpent-4-yn-1-one (3ha)

Light yellow liquid; yield: $38.3 \mathrm{mg}(57 \%)$.

IR (KBr): $1663 \mathrm{~cm}^{-1}$.

${ }^{1} \mathrm{H}$ NMR $\left(400 \mathrm{MHz}, \mathrm{CDCl}_{3}\right): \delta=7.60(\mathrm{~s}, 1 \mathrm{H}), 7.38-7.36(\mathrm{~m}, 2 \mathrm{H}), 7.27-$ $7.24(\mathrm{~m}, 4 \mathrm{H}), 6.56-6.54(\mathrm{~m}, 1 \mathrm{H}), 3.17(\mathrm{t}, J=7.2 \mathrm{~Hz}, 2 \mathrm{H}), 2.83(\mathrm{t}$, $J=7.2 \mathrm{~Hz}, 2 \mathrm{H})$.

${ }^{13} \mathrm{C}$ NMR $\left(100 \mathrm{MHz}, \mathrm{CDCl}_{3}\right): \delta=187.2,152.5,146.5,131.6,128.2$, 127.7, 123.6, 117.3, 112.3, 88.4, 81.2, 37.5, 14.2.

LR-MS (EI, $70 \mathrm{eV}): m / z(\%)=224\left(\mathrm{M}^{+}, 54\right), 223$ (100), 181 (75), 167 (64), 128 (54), 95 (63).

HRMS (ESI): $m / z[M+H]^{+}$calcd for $\mathrm{C}_{15} \mathrm{H}_{13} \mathrm{O}_{2}$ : 225.0910; found: 225.0917.

1-(4-Methoxyphenyl)-3-(phenylethynyl)octan-1-one (3ia)

Colorless liquid; yield: $50.1 \mathrm{mg}$ (50\%).

IR (KBr): $1680 \mathrm{~cm}^{-1}$.

${ }^{1} \mathrm{H} \mathrm{NMR}\left(400 \mathrm{MHz}, \mathrm{CDCl}_{3}\right): \delta=7.99(\mathrm{~d}, J=8.8 \mathrm{~Hz}, 2 \mathrm{H}), 7.34-7.31(\mathrm{~m}, 2$ $\mathrm{H}), 7.26-7.24(\mathrm{~m}, 3 \mathrm{H}), 6.94(\mathrm{~d}, J=8.8 \mathrm{~Hz}, 2 \mathrm{H}), 3.87(\mathrm{~s}, 3 \mathrm{H}), 3.32-3.26$ (m, $2 \mathrm{H}), 3.11-3.04(\mathrm{~m}, 1 \mathrm{H}), 1.64-1.61(\mathrm{~m}, 1 \mathrm{H}), 1.56-1.52(\mathrm{~m}, 1 \mathrm{H})$, $1.39-1.23(\mathrm{~m}, 6 \mathrm{H}), 0.92-0.86(\mathrm{~m}, 3 \mathrm{H})$.

${ }^{13} \mathrm{C}$ NMR $\left(100 \mathrm{MHz}, \mathrm{CDCl}_{3}\right): \delta=196.7,163.5,131.6,130.5,130.2$, $128.1,127.5,123.8,113.7,92.7,81.8,55.5,43.6,34.9,31.6,28.2,27.1$, $22.6,14.0$

LR-MS (EI, $70 \mathrm{eV}): m / z(\%)=334\left(\mathrm{M}^{+}, 4\right), 263$ (83), $215(23), 135$ (100). HRMS (ESI): $m / z[M+H]^{+}$calcd for $\mathrm{C}_{23} \mathrm{H}_{27} \mathrm{O}_{2}$ : 335.2006; found: 335.2011.

\section{1,7-Diphenylhept-6-yn-3-one (3ja)}

White solid; yield: $48.0 \mathrm{mg}(61 \%) ; \mathrm{mp} 63.4-64.8^{\circ} \mathrm{C}$.

IR (KBr): $1690 \mathrm{~cm}^{-1}$.

${ }^{1} \mathrm{H} \mathrm{NMR}\left(400 \mathrm{MHz}, \mathrm{CDCl}_{3}\right): \delta=7.37-7.34(\mathrm{~m}, 2 \mathrm{H}), 7.28-7.25(\mathrm{~m}, 5 \mathrm{H})$, $7.20-7.16(\mathrm{~m}, 3 \mathrm{H}), 2.92(\mathrm{t}, J=7.6 \mathrm{~Hz}, 2 \mathrm{H}), 2.78(\mathrm{t}, J=7.6 \mathrm{~Hz}, 2 \mathrm{H})$, $2.72-2.63(\mathrm{~m}, 4 \mathrm{H})$.

${ }^{13} \mathrm{C}$ NMR $\left(100 \mathrm{MHz}, \mathrm{CDCl}_{3}\right): \delta=207.8,140.8,131.5,128.5,128.3$, $128.2,127.7,126.1,123.5,88.5,81.0,44.3,41.7,29.6,13.9$.

LR-MS (EI, $70 \mathrm{eV}): m / z(\%)=262\left(\mathrm{M}^{+}, 14\right), 171$ (60), 157 (100), 105 (92), 91 (82).

HRMS (ESI): $m / z[M+H]^{+}$calcd for $\mathrm{C}_{19} \mathrm{H}_{19} \mathrm{O}$ : 263.1430; found: 263.1438 


\section{1-Phenylundec-1-yn-5-one (3ka)}

Light yellow liquid; yield: $52.3 \mathrm{mg}$ (72\%).

IR (KBr): $1710 \mathrm{~cm}^{-1}$.

${ }^{1} \mathrm{H}$ NMR $\left(500 \mathrm{MHz}, \mathrm{CDCl}_{3}\right): \delta=7.38-7.36(\mathrm{~m}, 2 \mathrm{H}), 7.27-7.26(\mathrm{~m}, 3 \mathrm{H})$, 2.74-2.65 (m, $4 \mathrm{H}$ ), 2.45 (t, J = 7.5 Hz, $2 \mathrm{H}$ ), 1.61-1.57 (m, $2 \mathrm{H}), 1.30-$ $1.26(\mathrm{~m}, 6 \mathrm{H}), 0.89-0.86(\mathrm{~m}, 3 \mathrm{H})$.

${ }^{13} \mathrm{C}$ NMR $\left(100 \mathrm{MHz}, \mathrm{CDCl}_{3}\right): \delta=209.0,131.5,128.1,127.6,123.6,88.6$, 80.8, 42.8, 41.4, 31.5, 28.8, 23.7, 22.4, 13.9 .

LR-MS (EI, $70 \mathrm{eV}): m / z(\%)=242\left(\mathrm{M}^{+}, 14\right), 171$ (16), 157 (100), 129 (27), 115 (35).

HRMS (ESI): $m / z[M+H]^{+}$calcd for $\mathrm{C}_{17} \mathrm{H}_{23} \mathrm{O}$ : 243.1743; found: 243.1747.

\section{1-(Adamantan-1-yl)-5-phenylpent-4-yn-1-one (3la)}

Colorless liquid; yield: $67.5 \mathrm{mg}$ (77\%).

IR (KBr): $1700 \mathrm{~cm}^{-1}$.

${ }^{1} \mathrm{H}$ NMR $\left(400 \mathrm{MHz}, \mathrm{CDCl}_{3}\right): \delta=7.38-7.36(\mathrm{~m}, 2 \mathrm{H}), 7.27-7.25(\mathrm{~m}, 3 \mathrm{H})$, 2.80-2.77 (m, 2 H), 2.65-2.61 (m, 2 H), 2.07-2.02 (m, 3 H), 1.85-1.82 (m, $5 \mathrm{H}), 1.81-1.68(\mathrm{~m}, 7 \mathrm{H})$.

${ }^{13} \mathrm{C}$ NMR $\left(100 \mathrm{MHz}, \mathrm{CDCl}_{3}\right): \delta=213.4,131.5,128.1,127.6,123.7,89.3$, 80.7, 46.2, 38.1, 36.5, 35.5, 27.9, 14.0.

LR-MS (EI, $70 \mathrm{eV}): m / z(\%)=292\left(\mathrm{M}^{+}, 5\right), 157$ (7), 135 (100).

HRMS (ESI): $m / z[M+H]^{+}$calcd for $\mathrm{C}_{21} \mathrm{H}_{25} \mathrm{O}$ : 293.1900; found: 293.1908.

\section{(E)-1,7-Diphenylhept-1-en-6-yn-3-one (3ma)}

Yellow solid; yield: $42.9 \mathrm{mg}(55 \%) ; \mathrm{mp} 46.7-47.9^{\circ} \mathrm{C}$.

IR (KBr): $1696 \mathrm{~cm}^{-1}$.

${ }^{1} \mathrm{H} \mathrm{NMR}\left(400 \mathrm{MHz}, \mathrm{CDCl}_{3}\right): \delta=7.61(\mathrm{~d}, J=16.4 \mathrm{~Hz}, 1 \mathrm{H}), 7.57-7.55(\mathrm{~m}$, $2 \mathrm{H}), 7.41-7.37(\mathrm{~m}, 5 \mathrm{H}), 7.27-7.26(\mathrm{~m}, 3 \mathrm{H}), 6.78(\mathrm{~d}, J=16.0 \mathrm{~Hz}, 1 \mathrm{H})$, 3.04-3.01 (m, $2 \mathrm{H}), 2.81-2.77(\mathrm{~m}, 2 \mathrm{H})$.

${ }^{13} \mathrm{C}$ NMR $\left(100 \mathrm{MHz}, \mathrm{CDCl}_{3}\right): \delta=198.1,143.1,134.4,131.6,130.6$, 129.0, 128.3, 128.2, 127.7, 125.9, 123.6, 88.8, 81.1, 39.7, 14.3.

LR-MS (EI, $70 \mathrm{eV}): m / z(\%)=260\left(\mathrm{M}^{+}, 4\right), 217(81), 203(42), 131$ (56), $103(100)$.

HRMS (ESI): $m / z[M+H]^{+}$calcd for $\mathrm{C}_{19} \mathrm{H}_{17} \mathrm{O}: 261.1274$; found: 261.1280 .

\section{Acknowledgment}

We thank the Natural Science Foundation of China (Nos. 21472039 and 21172060) and Hunan Provincial Natural Science Foundation of China (No. 13JJ2018) for financial support.

\section{Supporting Information}

Supporting information for this article is available online at http://dx.doi.org/10.1055/s-0035-1560374.

\section{Reference}

(1) For selected reviews and papers: (a) Patai, S. The Chemistry of Triple-Bonded Functional Groups; Wiley: New York, 1994. (b) Stang, P. J.; Diederich, F. Modern Acetylene Chemistry; VCH: Weinheim, 1995. (c) Diederich, F.; Stang, P. J.; Tykwinski, R. R. Acetylene Chemistry: Chemistry, Biology and Material Science; Wiley-VCH: Weinheim, 2005.

(2) (a) Negishi, E.; Anastasia, L. Chem. Rev. 2003, 103, 1979. (b) Tykwinski, R. R. Angew. Chem. Int. Ed. 2003, 42, 1566. (c) Plenio, H. Angew. Chem. Int. Ed. 2008, 47, 6954. (d) Chinchilla, R.; Nájera, C. Chem. Rev. 2007, 107, 874. (e) de Meijere, A.; Diederich, F. Metal-Catalyzed Cross-Coupling Reactions, 2nd ed.; Wiley-VCH: Weinheim, 2004. (f) Doucet, H.; Hierso, J. Angew. Chem. Int. Ed. 2007, 46, 834.

(3) (a) Eckhardt, M.; Fu, G. C. J. Am. Chem. Soc. 2003, 125, 13642. (b) Vechorkin, O.; Barmaz, D.; Proust, V.; Hu, X. J. Am. Chem. Soc. 2009, 131, 12078. (c) Garcia, P. M. P.; Ren, P.; Scopelliti, R.; Hu, X. ACS Catal. 2015, 5, 1164. (d) Hatakeyama, T.; Okada, Y.; Yoshimoto, Y.; Nakamura, M. Angew. Chem. Int. Ed. 2011, 50, 10973. (e) Vechorkin, O.; Godinat, A.; Scopelliti, R.; Hu, X. Angew. Chem. Int. Ed. 2011, 50, 11777. (f) Cheung, C. W.; Ren, P.; $\mathrm{Hu}, \mathrm{X}$. Org. Lett. 2014, 16, 2566. (g) Xu, T.; Hu, X. Angew. Chem. Int. Ed. 2015, 54, 1307.

(4) (a) Ouyang, X.-H.; Song, R.-J.; Wang, C.-Y.; Yang, Y.; Li, J.-H. Chem. Commun. 2015, 51, 14497. (b) Wang, Z.; Li, L.; Huang, Y. J. Am. Chem. Soc. 2014, 136, 12233. (c) Wang, Z.; Li, X.; Huang, Y. Angew. Chem. Int. Ed. 2013, 52, 14219. (d) Wang, H.; Xie, F.; Qi, Z.; Li, X. Org. Lett. 2015, 17, 920.

(5) (a) Li, Y.; Liu, X.; Jiang, H.; Liu, B.; Chen, Z.; Zhou, P. Angew. Chem. Int. Ed. 2011, 50, 6341. (b) Nicolai, S.; Piemontesi, C.; Waser, J. Angew. Chem. Int. Ed. 2011, 50, 4680. (c) González, D. F.; Brand, J. P.; Waser, J. Chem. Eur. J. 2010, 16, 9457. (d) Brand, J. P.; Waser, J. Chem. Soc. Rev. 2012, 41, 4165. (e) Vaillant, F. L.; Courant, T.; Waser, J. Angew. Chem. Int. Ed. 2015, 54, 11200. (f) Yang, J.; Zhang, J.; Qi, L.; Hu, C.; Chen, Y. Chem. Commun. 2015, 51, 5275. (g) Feng, Y.-S.; Xu, Z.-Q.; Mao, L.; Zhang, F.-F.; Xu, H.-J. Org. Lett. 2013, 15, 1472. (h) Yang, Y.; Huang, H.; Zhang, X.; Zeng, W.; Liang, Y. Synthesis 2013, 45, 3137. (i) Finkbeiner, P.; Weckenmann, N. M.; Nachtsheim, B. J. Org. Lett. 2014, 16, 1326. (j) Huang, H.; Zhang, G.; Gong, L.; Zhang, S.; Chen, Y.J. Am. Chem. Soc. 2014, 136, 2280. (k) Wen, Y.; Wang, A.; Jiang, H.; Zhu, S.; Huang, L. Tetrahedron Lett. 2011, 52, 5736. (1) Li, Y.; Liu, X.; Jiang, H.; Feng, Z. Angew. Chem. Int. Ed. 2010, 49, 3338.

(6) (a) Fujiwara, Y.; Domingo, V.; Seiple, I. B.; Gianatassio, R.; Del Bel, M.; Baran, P. S. J. Am. Chem. Soc. 2011, 133, 3292. (b) Seiple, I. B.; Su, S.; Rodriguez, R. A.; Gianatassio, R.; Fujiwara, Y.; Sobel, A. L.; Baran, P. S. J. Am. Chem. Soc. 2010, 132, 13194. (c) Lockner, J. W.; Dixon, D. D.; Risgaard, R.; Baran, P. S. Org. Lett. 2011, 13, 5628. (d) Hu, F.; Shao, X.; Zhu, D.; Lu, L.; Shen, Q. Angew. Chem. Int. Ed. 2014, 53, 6105. (e) Liu, X.; Wang, Z.; Cheng, X.; Li, C. J. Am. Chem. Soc. 2012, 134, 14330. (f) Wang, H.; Guo, L.-N.; Duan, X.-H. Adv. Synth. Catal. 2013, 355, 2222. (g) Wang, P.-F.; Wang, X.-Q.; Dai, J.-J.; Feng, Y.-S.; Xu, H.-J. Org. Lett. 2014, 16, 4586. (h) Mai, W.-P.; Sun, G.-C.; Wang, J.-T.; Song, G.; Mao, P.; Yang, L.-R.; Yuan, J.-W.; Xiao, Y.-M.; Qu, L.-B. J. Org. Chem. 2014, 79, 8094.

(7) (a) Li, Y.; Wang, J.; Wei, X.; Yang, S. Chin. J. Org. Chem. 2015, 35 , 638. (b) Bloom, S.; Bume, D. D.; Pitts, C. R.; Lectka, T. Chem. Eur. J. 2015, 21, 8060. (c) Li, Y.; Ye, Z.; Bellman, T. M.; Chi, T.; Dai, M. Org. Lett. 2015, 17, 2186. (d) Ye, Z.; Dai, M. Org. Lett. 2015, 17, 
Synthesis

2190. (e) Zhao, H.; Fan, X.; Yu, J.; Zhu, C. J. Am. Chem. Soc. 2015 , 137, 3490. (f) Jiao, J.; Nguyen, L. X.; Patterson, D. R.; Flowers, R. A. II Org. Lett. 2007, 9, 1323. (g) Ilangovan, A.; Saravanakumar, S.; Malayappasamy, S. Org. Lett. 2013, 15, 4968. (h) Wang, Y. F.; Chiba, S. J. Am. Chem. Soc. 2009, 131, 12570. During our prepare for this paper, a very similar report has come out. In this paper, excess amount of $\mathrm{AcOH}$ was required to promote the reaction with cyclopropanols. Furthermore, the scope is limited to silyland phenyl-substituted alkynes, see: (i) Wang, S.; Guo, L. N.; Wang, H.; Duan, X. H. Org. Lett. 2015, 17, 4798.

(8) (a) Ishida, K.; Kusama, H.; Iwasawa, N. J. Am. Chem. Soc. 2010, 132, 8842. (b) Cai, Y.; Jalan, A.; Kubosumi, A. R.; Castle, S. L. Org. Lett. 2015, 17, 488. (c) Imagawa, H.; Kurisaki, T.; Nishizawa, M. Org. Lett. 2004, 6, 3679.

(9) Zheng, H. C.; Felix, R. J.; Gagné, M. R. Org. Lett. 2014, 16, 2272. 